\title{
GROUND STATE AND THERMODYNAMIC PROPERTIES OF AN INDUCED-PAIRING MODEL
}

\author{
G. PaWeowski and S. RobaszKIEWICZ \\ Institute of Physics, A. Mickiewicz University \\ Umultowska 85, 61-614 Poznań, Poland
}

(Received July 9, 1998)

\begin{abstract}
We study the phase diagrams and thermodynamic properties of a system of coexisting wide-band current carriers (c-particles) and narrow-band electrons ( $d$-electrons) which can form local pairs. There are two distinct mechanisms of superconductivity in the model considered: (i) the intersubsystem charge exchange, which leads to the superconducting state involving both types of electrons and (ii) the pair hopping interaction of $d$-electrons, leading to the superconducting state of $d$-particles only. In contrast to previous works on the subject, we assume an arbitrary value of on-site density interaction of $d$-electrons $U$, which allows us to study the effects of reduced $d$-pair binding energy. Within the approach in which the $U$ term is exactly treated we determine the evolution of superconducting properties as a function of interactions and relative position of the bands. In a definite range of parameters the system shows features which are intermediate between those of a local pair superconductor and those of a classical BCS model. The increasing on-site Coulomb repulsion $U$ competing with the intersubsystem charge exchange and the pair hopping interaction reduces the superconducting critical temperature. Moreover, it can induce a change of the superconducting transition into the first order one. Above a critical value of $U$, dependent on concentration of electrons and other interactions, the superconducting state cannot be stable at any temperature.
\end{abstract}

PACS numbers: 74.20.-z, 71.28.+d, 74.25.Ha

\section{Introduction}

The coexistence of real-space bound pairs and itinerant electrons as well as the effects resulting from interactions between these two species constitute a crucial problem for real materials. A model of a mixture of local pairs and wide band electrons was introduced several years ago $[1,2]$ for a unified description of nonconventional superconductors, chalcogenide glasses, nonsimple metals and the systems with alternating valency. 
Its various versions have been extensively analysed in numerous papers [3-10] and several attempts checking its applicability to high-temperature superconductors have been made. It has been shown that the model is capable of explaining several anomalous properties of these materials which are in conflict with BCS theory or its strong coupling extensions [3,6-10].

In all the above mentioned works the binding energy of local pairs was assumed to be very high which allowed the pairs to be treated as (hard-core) bosons with a charge $2 e$, and the model could be called the boson-fermion model.

Obviously, such an assumption simplifies analyses but also restricts the range of applicability of the obtained results.

For this reason we will discuss a generalization of the above model, i.e. we shall consider the model for an assumed arbitrary value of on-site interaction of localised electrons $U$, which makes it possible to study the effects of reduced pair-binding energy.

The considered effective Hamiltonian of coexisting localized $d$-electrons and itinerant $c$-electrons can be written as:

$$
H=H_{d}+H_{c}+H_{c d}
$$

where

$$
\begin{aligned}
& H_{d}=\sum_{i}\left(E_{0}-\mu\right) n_{i}^{d}+U \sum_{i} n_{i \uparrow}^{d} n_{i \downarrow}^{d}-\frac{1}{2} \sum_{i j} J_{i j} d_{i \uparrow}^{+} d_{i \downarrow}^{+} d_{j \downarrow} d_{j \uparrow}+\text { h.c. } \\
& H_{c}=\sum_{k \sigma}\left(\varepsilon_{k}-\mu+\varepsilon_{0}\right) c_{k \sigma}^{+} c_{k \sigma} \\
& H_{c d}=\frac{I_{0}}{2 N} \sum_{i k k^{\prime}}\left(\mathrm{e}^{\mathrm{i}\left(k-k^{\prime}\right) R_{i}} c_{k \uparrow}^{+} c_{k^{\prime} \downarrow}^{+} d_{i \downarrow} d_{i \uparrow}+\text { h.c. }\right) \\
& n_{i}^{d}=\sum_{\sigma} d_{i \sigma}^{+} d_{i \sigma}
\end{aligned}
$$

where $E_{0}$ measures the relative position of $d$-level with respect to the bottom of the $c$-electron band $\varepsilon_{k}$ in the absence of interactions, $\mu$ is the chemical potential which ensures that a total number of particles in the system is constant, i.e.

$$
n=n_{c}+n_{d}=\frac{1}{N}\left(\sum_{k \sigma}\left\langle c_{k \sigma}^{+} c_{k \sigma}\right\rangle+\sum_{i}\left\langle n_{i}^{d}\right\rangle\right),
$$

$U$ is the effective on-site density interaction between $d$-electrons, $J_{i j}$ is the intersite charge exchange interaction of $d$-electrons (pair hopping). The term proportional to $I_{0}$ represents the transverse component of the charge exchange between the two subsystems (which is crucial for the superconducting mechanism in the system considered). To simplify our analysis we do not include in Eq. (1) the single electron hopping term for $d$ electrons as well as other intersite interaction terms.

The interactions $U, I_{0}$ and $J_{i j}$ will be treated as effective ones and will be assumed to include all the possible contributions and renormalizations like those coming from the strong electron-phonon (exciton) coupling or from the coupling between the electrons considered and other electronic subsystems in solid or chemical complexes $[2,3]$. 
In the absence of " $c$ " subsystem the problem reduces to the one of the on-site pairs with finite binding energy analysed in Ref. [11]. In the limit of $U \rightarrow-\infty$ we recover the extensively studied model of coexisting itinerant electrons and hard-core charged bosons on a lattice (Refs. [1-3, 6-9]). Preliminary results concerning the case $J_{i j}=0$ have been already presented in Ref. [12].

\section{Free energy and self-consistent equations}

In analysis of model (1) we use the variational approach which treats the on-site interaction term $U$ exactly (as in Ref. [11]) and the intersite and intersubsystem interactions within the broken symmetry Hartree-Fock approximation (as in Ref. [2]). (SS) is

The resulting expression for the free energy of the superconducting phase

$$
\frac{F_{0}}{N}=-\frac{2}{\beta N} \sum_{k} \ln \left(2 \cosh \frac{\beta A_{k}}{2}\right)-\frac{1}{\beta} \ln 2 Z_{d}+\mu\left(n_{d}+n_{c}\right)-\widetilde{\mu}-\bar{\mu}+C,
$$

where

$$
\begin{aligned}
& C=J_{0}\left(\rho_{0}^{x}\right)^{2}+2\left|I_{0}\right| x_{0} \rho_{0}^{x}, \quad A_{k}=\left[\left(\varepsilon_{k}-\bar{\mu}\right)^{2}+\left(I_{0} \rho_{0}^{x}\right)^{2}\right]^{1 / 2}, \\
& Z_{d}=\exp \left(\beta \frac{U}{2}\right)+\cosh \beta \Delta, \quad \Delta=\sqrt{\tilde{\mu}^{2}+\left(J_{0} \rho_{0}^{x}+\left|I_{0}\right| x_{0}\right)^{2}} \\
& \bar{\mu}=\mu-\varepsilon_{0}, \quad \tilde{\mu}=\mu-E_{0}-U / 2, \quad J_{0}=\sum_{j \neq i} J_{i j}, \quad \beta=\left(k_{\mathrm{B}} T\right)^{-1}, \\
& \rho_{0}^{x}=\frac{1}{N} \sum_{i}\left\langle d_{i \uparrow}^{+} d_{i \downarrow}^{+}\right\rangle, \quad \text { and } \quad x_{0}=\frac{1}{N} \sum_{k}\left\langle c_{k \uparrow}^{+} c_{-k \downarrow}^{+}\right\rangle
\end{aligned}
$$

are the superconducting order parameters for $d$ and $c$ subsystems, respectively, and $E_{\mathrm{g}}(T)=\min 2 A_{k}$ is the energy gap in the $c$-electron spectrum.

Upon minimizing this free energy with respect to the variables $\rho_{0}^{x}, x_{0}$ and $\mu$ we obtain the following self-consistent equations:

$$
\begin{aligned}
& x_{0}=\frac{1}{2 N} \sum_{k} \frac{\left|I_{0}\right| \rho_{0}^{x}}{A_{k}} \tanh \frac{\beta A_{k}}{2}, \\
& \rho_{0}^{x}=\left(J_{0} \rho_{0}^{x}+\left|I_{0}\right| x_{0}\right) \frac{\sinh \beta \Delta}{2 \Delta Z_{d}} \\
& n=n_{c}+n_{d},
\end{aligned}
$$

where $n_{c}$ and $n_{d}$ are given by

$$
n_{c}-1=\frac{1}{N} \sum_{k} \frac{\varepsilon_{k}-\bar{\mu}}{A_{k}} \tanh \frac{\beta A_{k}}{2}, \quad n_{d}-1=\frac{\tilde{\mu} \sinh \beta \Delta}{2 Z_{d}}
$$

and $n$ is the total number of particles ( $c$ and $d$ ) per site. 
From $F_{0}$ one can obtain the local magnetic moment of $d$-electrons $\gamma_{d}$. This quantity is defined by

$$
\gamma_{d}=\frac{1}{N} \sum_{i}\left\langle\left|n_{i \uparrow}^{d}-n_{i \downarrow}^{d}\right|\right\rangle=n_{d}-\frac{2}{N} \sum_{i}\left\langle\left|n_{i \uparrow}^{d} n_{i \downarrow}^{d}\right|\right\rangle=n-2\left(\frac{\partial F_{0} / N}{\partial U}\right)_{V, T},
$$

and it is directly related to the concentration of paired $d$-electrons $n_{p}^{d}$ :

$$
n_{p}^{d}=n-\gamma_{d}=\frac{2}{N}\left(\frac{\partial F_{0} / N}{\partial U}\right)_{V, T} .
$$

$N \gamma_{d}$. and $N n_{p}^{d}$ determine the number of sites singly and doubly occupied by $d$-electrons, respectively.

We have performed a quite extended analytical and numerical analysis of Eqs. (3)-(5), both at $T=0$ and for $T>0$, and examined the phase diagrams and thermodynamic properties of the system as a function of $E_{0}, n$ and various coupling constants. In the calculations we have made use of the rectangular density of states (DOS) for $c$-electrons:

$$
N(\varepsilon)= \begin{cases}1 /(2 D), & \text { for } 0 \leq \varepsilon_{k}+\varepsilon_{0} \leq 2 D, \\ 0, & \text { otherwise }\end{cases}
$$

with $D$ denoting an effective half-bandwidth, and assumed the bottom of the wide band to have zero energy, i.e. $\min \left(\varepsilon_{k}+\varepsilon_{0}\right)=0$.

Several analytical results concerning the ground state characteristics and the critical temperatures, which can be derived in particular limits, are collected in Appendix for comparison with numerical solutions.

In order to appreciate the difference in the superconducting properties due to the two distinct mechanisms involved here $\left(J_{0}\right.$ and $\left.I_{0}\right)$, we shall discuss the two cases: $J_{0} \neq 0, I_{0}=0$ and $J_{0}=0, I_{0} \neq 0$ separately.

\section{The case of $I_{k k^{\prime}}=0, J_{i j} \neq 0$}

For $I_{0}=0, J_{0} \neq 0$ the SS state can develop only in the $d$-electron subsystem, and its properties are similar to those of the one band model studied in Ref. [11]. A difference in comparison to [11] is that in the present case the number of superconducting $d$-electrons is no longer a constant, but will vary as a function of temperature, so that both $d$ and $c$ electrons are in thermal equilibrium and their total number $n$ is constant.

For $I_{0}=0$ self-consistent equations (5) for $\Delta$ and $n$ take the form

$$
\begin{aligned}
& \Delta=J_{0} \frac{\sinh \beta \Delta}{2 Z_{d}}, \\
& n=n_{c}+n_{d},
\end{aligned}
$$

where

$$
\begin{aligned}
& n_{c}-1=-\frac{1}{N} \sum_{k} \tanh \beta \frac{\varepsilon_{k}-\bar{\mu}}{2}, \quad n_{d}-1=\frac{\left(\widetilde{\mu}-\Delta_{0}\right) \sinh \beta \Delta}{\Delta Z_{d}}, \\
& \Delta=\sqrt{\left(\tilde{\mu}-\Delta_{0}\right)^{2}+\left[J_{0} \rho_{0}^{x}\right]^{2}}, \quad \Delta_{0}=E_{0}+U / 2,
\end{aligned}
$$


and the explicit expressions determining the concentration of paired $d$-electrons in SS and nonordered (NO) phases (Eq. (7)) are

$$
\begin{aligned}
& n_{p}^{d}(\mathrm{SS})=n_{d}-\frac{\exp (\beta U / 2)}{\exp (\beta U / 2)+\cosh \beta J_{0} \sqrt{\left[(1 / 2)\left(n_{d}-1\right)\right]^{2}+\left(\rho_{0}^{x}\right)^{2}}}, \\
& n_{p}^{d}(\mathrm{NO})=n_{d}-\frac{2 \exp \beta(\widetilde{\mu}+U / 2)}{1+2 \exp \beta(\widetilde{\mu}+U / 2)+2 \exp \beta \widetilde{\mu}} .
\end{aligned}
$$

The transition from the superconducting phase $\left(\rho_{0}^{x} \neq 0\right)$ to the normal state $\left(\rho_{0}^{x}=0\right)$ can be continuous (2nd order) or discontinuous (1st order), depending on the values of $U$ and $n_{d}$.

The second-order transition temperature $T_{\mathrm{c}}$ is determined by the equation

$$
\frac{U}{J_{0}}=\frac{2}{\beta_{\mathrm{c}} J_{0}} \ln \left[\frac{1}{\left|n_{d}-1\right|} \sinh \beta_{\mathrm{c}} J_{0} \frac{\left|n_{d}-1\right|}{2}-\cosh \beta_{\mathrm{c}} J_{0} \frac{\left|n_{d}-1\right|}{2}\right]
$$

and Eqs. (10), (11) taken in the limit $\rho_{0}^{x} \rightarrow 0$.

It can be shown that the phase transition remains continuous for arbitrary $U \leq 0$. This is due to the fact that $U$ and $J_{0}\left(I_{0}\right)$ are cooperating interactions for attractive $U$.

For $U \leq 0$ the ground state phase diagrams as a function of $n$ vs. $\Delta_{0} / D$ are shown in Fig. 1.
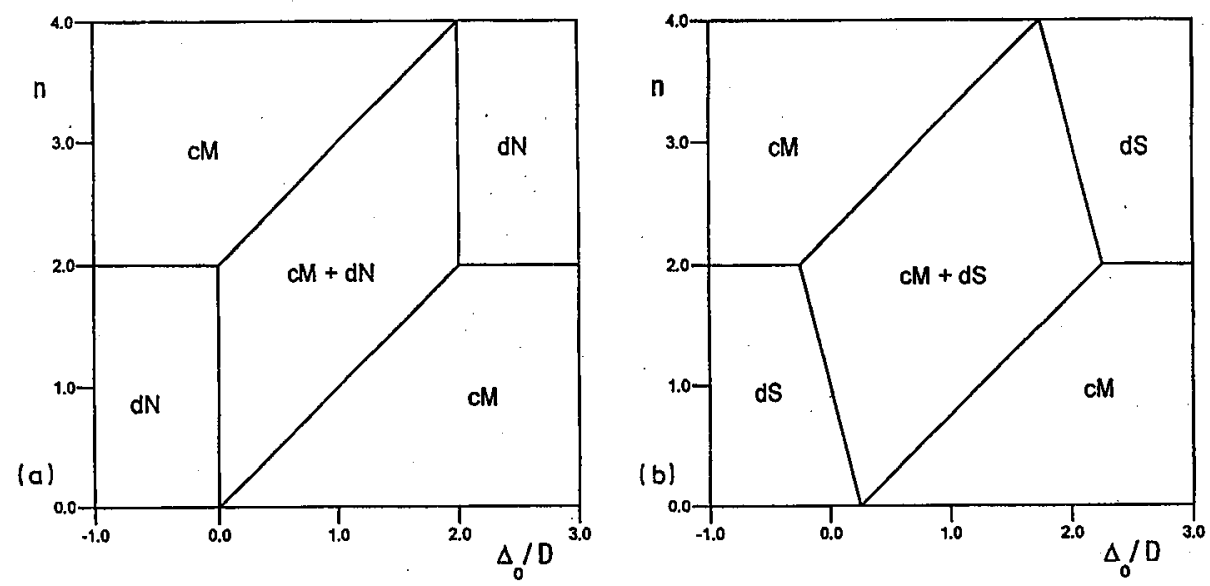

Fig. 1. The ground state phase diagram as a function of $n$ versus $\left(E_{0}+U / 2\right) / D=$ $\Delta_{0} / D(-\infty \leq U \leq 0)$ for (a) $J_{0} \stackrel{\circ}{=} 0, I_{0}=0$ and (b) $J_{0} / 2 D=0.25, I_{0}=0$. Notation: $c M$ is metallic state of $c$-electrons, $d N$ is nonmetallic state of $d$-electrons, $d S$ is superconducting (SS) state of $d$-electrons.

The regions of superconducting state for $n \leq 2$ are determined by

$$
-\frac{J_{0}}{2 D}(n-1)<\left(E_{0}+\frac{U}{2}\right) / D<\frac{J_{0}}{2 D}+n .
$$

For any $U \leq 0$ the transition at $E_{0}+U / 2=J_{0} / 2+n D$ between the SS state with $n_{d} \neq 0$ and the nonordered state with $n_{c}=n$ is always of second order. The same 
is true for $n=2$ at $\Delta_{0}=-J_{0} / 2$ between the SS phase and the nonordered one with $n_{d}=2$. On the other hand, the transition for $n<2$ at $\Delta_{0}=-(n-1)\left(J_{0} / 2\right)$ between the SS phase with $n_{d}=n$ and the SS phase with $n_{d}<n, n_{c}>0$ is always of higher order.

In Fig. 2 we plot the finite temperature phase diagrams as a function of $\Delta_{0} / D$ for fixed values of $n$ and $J_{0} / 2 D(U \rightarrow-\infty$ and $U=0)$. Only for $n=2$ the SS region is well confined between the two limiting values of $\Delta_{0} / D: J_{0} / 2<\Delta_{0}<J_{0} / 2+2 D$. For an arbitrary $n<2$, on the contrary, the SS phase extends over the entire region of $\Delta_{0}<J_{0} / 2+n D$ for sufficiently low temperatures.

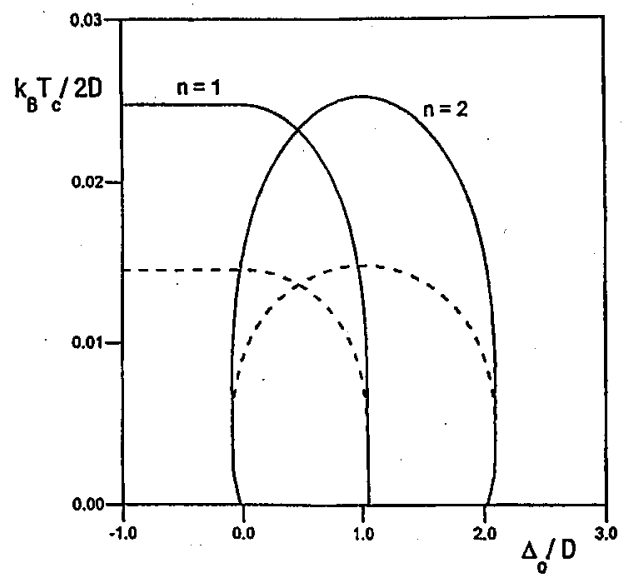

Fig. 2. The superconducting critical temperature $T_{c}$ as a function of $\Delta_{0} / D$ for two values of $n$ (=2 and 1), $J_{0} / 2 D=0.05, I_{0}=0 . U / D \rightarrow-\infty$ (solid), $U=0$ (dashed).

A general structure of the phase diagrams remains qualitatively the same for any $U \leq 0$. For any fixed $n$ the transition temperatures increase with increasing $|U|(U<0)$ and $T_{\mathrm{c}}(U \rightarrow-\infty) \approx 2 T_{\mathrm{c}}(U=0)$, if $J_{0} / 2 D \ll 1$.

The effect of repulsive $U$ is much more drastic as it competes with $J_{0}$ and destabilizes superconductivity. As we shall see below, increasing $U>0$ changes first the nature of the phase transition from a continuous to a discontinuous type, leading to the appearance of a tricritical point (TCP), then it suppresses superconductivity for high $n_{d}\left(\left|n_{d}-1\right| \approx 0\right)$. Finally, for large $U\left(U / J_{0}>1\right)$, the system remains in the normal state for all $T$ and $n_{d}$.

The TCP, where the transition changes into the first order one, is given by the equation:

$$
\tanh \left[\beta_{\mathrm{TCP}} J_{0} \frac{\left|n_{d}-1\right|}{2}\right]=\left[\frac{2}{\left|n_{d}-1\right| \beta_{\mathrm{TCP}} J_{0}}+\left|n_{d}-1\right|\right]^{-1}
$$

and Eqs. (13).

The critical temperatures for the first-order phase transition, necessary to construct a complete phase diagram have to be determined numerically by comparing the free energies of SS and NO phases. 
For simplicity the next two figures have been made for $J_{0} / 2 D \ll 1$. In such a case one can neglect the $T$ dependence of $n_{d}$ for any $n$ (if $k_{\mathrm{B}} T / D \ll 1$ ) and make the plots of $T_{\mathrm{c}}$ as a function of $n_{d}$. Taking into account that

$$
\begin{aligned}
& n_{d} \approx n_{d}(T=0)=\frac{-\Delta_{0}+n D+J_{0} / 2}{D+J_{0} / 2}, \\
& \quad \text { for }-\frac{J_{0}}{2 D}(n-1)<\frac{\Delta_{0}}{D}<\frac{J_{0}}{2 D}+n, \\
& n_{d}=n, \quad \text { for } \frac{\Delta_{0}}{D}<-\frac{J_{0}}{2 D}(n-1), \\
& n_{d}=0, \quad \text { for } \frac{\Delta_{0}}{D}>\frac{J_{0}}{2 D}+n,
\end{aligned}
$$

the obtained diagrams can be transformed directly into those plotted as a function of $\Delta_{0} / D$ for any $n$.

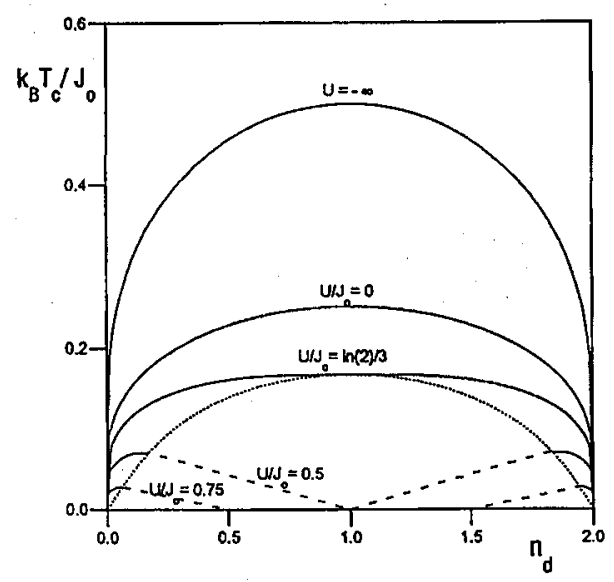

Fig. 3. $k_{\mathrm{B}} T_{c} / J_{0}$ vs. $n_{d}$ plotted for different values of $U / J_{0}$ (numbers to the curves). The second- and first-order transitions are indicated by the solid and dashed lines, respectively. Tricritical points are shown as dotted line. $J_{0} / 2 D=0.05, I_{0}=0$.

Figure 3 shows the $n_{d}$ dependence of the transition temperature for several different values of $U / J_{0}$. Between $U \rightarrow-\infty$ and $U / J_{0}=(1 / 3) \ln 2$ the $T_{\mathrm{c}}$ is maximum for $U \rightarrow-\infty, n_{d}=1$ and decreases monotonically with increasing $U$ and $\left|n_{d}-1\right|$. For $U / J_{0}>(1 / 3) \ln 2$ the $n_{d}$ dependence of $T_{c}$ becomes nonmonotonic and with increasing $U$ the $T_{c}(n)$ maximum moves towards lower $n_{d}$ (higher $\left.\left|n_{d}-1\right|\right)$. For $(1 / 3) \ln 2<U / J_{0}<0.5$ the ground state is SS for $0<n_{d}<2$, whereas for $0.5<U / J_{0}<1$ the $n_{d}$ range of the SS phase is reduced with increasing $U$.

The evolution of the concentration of paired $d$ electrons $n_{p}^{d}$ with temperature strongly depends on the $U / J_{0}$ value. This can be clearly seen from Fig. 4. One can single out two limiting types of the $n_{p}^{d}(T)$ behaviour. The first one is realised in the case of large on-site attraction (large $d$-pair binding energy: 


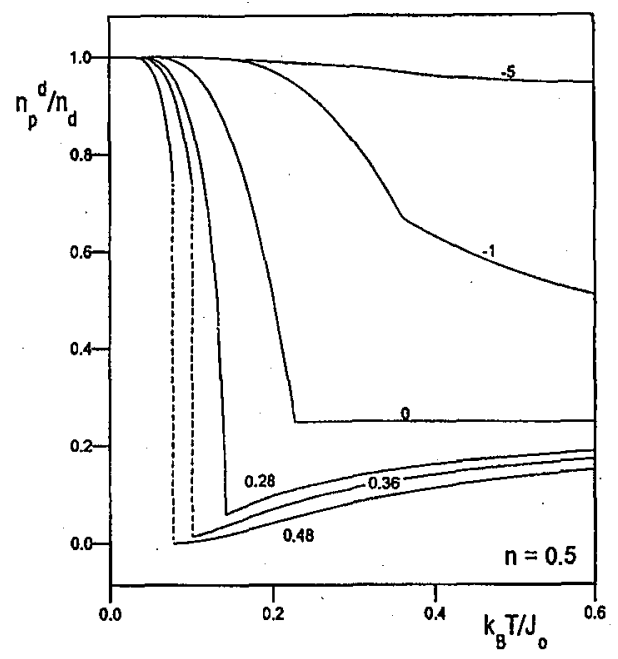

Fig. 4. Temperature dependence of the concentration of paired $d$-electrons $n_{d}^{p}$ (normalized to $n_{d}$ ) plotted for increasing values of $U / J_{0} . n_{d}=0.5, J_{0} / 2 D=0.05$.

$E_{\mathrm{b}}=-U+J_{0} \gg k_{\mathrm{B}} T_{\mathrm{c}}$ ), whereas the second one occurs if the binding energy becomes comparable to (or smaller than) $k_{\mathrm{B}} T_{\mathrm{c}}$. In between, there is a smooth crossover between the two regimes. In the first limit (local pair regime), $n_{p}^{d}$ changes slowly with temperature and the transition at $T_{\mathrm{c}}$ is to the state of dynamically disordered local pairs. In the second limit (pair breaking regime) $n_{p}^{d}$ exhibits a sharp break at $T_{\mathrm{c}}$ and a substantial fraction of single $d$ electrons exists above $T_{\mathrm{c}}$. As temperature is lowered, the condensate grows both from a condensation of preexisting $d$ pairs and from binding and condensation of single particles, and at $T=0$ we have $n_{p}^{d}=n_{d}$.

\section{The case of $J_{i j}=0, I_{k k^{\prime}} \neq 0$}

In the case of $J_{0}=0, I_{0} \neq 0$ superconductivity can only occur provided that both subsystems ( $d$ and $c$ ) participate in its formation. Depending on the relative concentration of " $c$ " and " $d$ " electrons we distinguish three essentially different physical situations $(0<n<2)$ :

(i) $d$-regime, $n_{d} \gg n_{c} \geq 0\left(n_{d} \approx n\right)$ :

Pairs of $d$-electrons move via virtual excitations into empty $c$-electrons states. Such a mechanism gives rise to the long-range hopping of $d$-pairs, of a spatial dependence similar to the RKKY interaction for $s-d$ mechanism in the magnetic equivalent (for $U \ll 0$ the explicit form of this long-range hopping can be derived from Eq. (1) by a unitary transformation reducing $H_{c-d}$ to the first order $\left.[2,3,6]\right)$.

(ii) $d+c$ (mixed valence) regime; $n_{c}, n_{d}>0$ :

SS comes about by a perpetual interchange between $d$ and $c$ pairs. In this process " $c$ " electrons become polarized into Cooper pairs and " $d$ " pairs increase their mobility by decaying into " $c$ " pairs. For this intermediate case neither the standard BCS picture nor the picture of local pairs strictly applies and superconductivity has a "mixed" character. 
(iii) c-regime $n_{c} \gg n_{d} \geq 0\left(n_{c} \approx n\right)$ :

The situation is similar to the BCS case: pairs of "c" electrons with opposite momenta and spins are exchanged via virtual transitions into empty $d$-levels.

\subsection{Properties of the system for $-\infty \leq U<0$}

Let us discuss the case $U \leq 0$ first.

At $T=0$ and $U \leq 0$ numerical solution of Eqs. (4), (5) shows that the SS state is always the ground state for all values of $I_{0} / D$ and $\left(E_{0}+U / 2\right) / D$ for any value of $n$ except $n=2$. For $n=2$ the ground state phase diagram is plotted in Fig. 5. In this case both normal phases ( $d N$ and $c N)$ are insulating and the phase boundaries with the SS state are of second order.

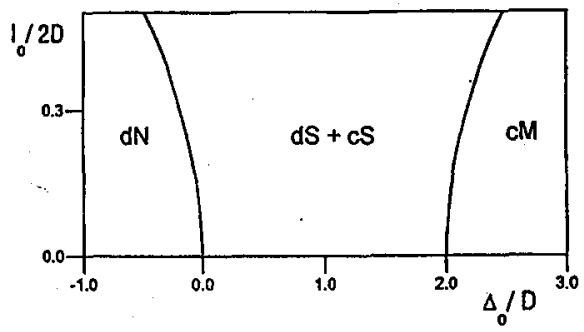

Fig. 5. The ground-state phase diagram for $n=2, J_{0}=0$ as a function of $I_{0} / 2 D$, vs. $\Delta_{0} / D\left(D_{0}=E_{0}+U / 2\right)(U \leq 0) \cdot d S+c S$ denotes the superconducting state involving both $d$ and c electrons. $d N$ and $c M$ indicate the nonmetallic states of $d$ and $c$ electrons, respectively.

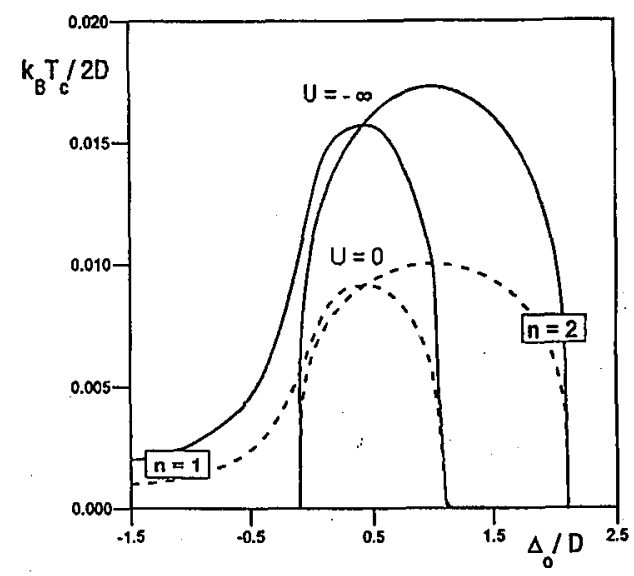

Fig. 6. The superconducting critical temperature $T_{\mathrm{c}}$ as a function of $\Delta_{0} / D$ plotted for two fixed values of $n$ and $I_{0} / 2 D=0.1$. $U \rightarrow-\infty$ (solid lines), $U=0$ (dashed lines).

In Fig. 6 the finite temperature phase diagrams as a function $\Delta_{0} / D$ are shown for the limits $U \rightarrow-\infty$ and $U=0$. 
In general, as we go from the case of predominantly $d$-electrons to that of predominantly wide band-electrons (i.e. with increasing $E_{0}$ ), we observe a non-monotonic behaviour of $T_{\mathfrak{c}}$, which passes through a maximum of an order of $I^{2} / D$, when the two constituents have roughly equal concentrations and drops to zero when we approach the regions (i) and (iii).

Figure 7 shows the variation of the ratio $2 k_{\mathrm{B}} T_{\mathrm{c}} / E_{\mathrm{g}}(0)$ as a function of $\Delta_{0} / D\left(E_{\mathrm{g}}(0)\right.$ is the gap in the $c$-electron excitation spectrum at $\left.T=0\right)$. As we see the inverse of the ratio varies around the BCS value 3.52 as the relative proportion of $d$-electrons to wide band electrons is changed. Where $T_{\mathrm{c}}$ is maximum $E_{\mathrm{g}}(0) / k_{\mathrm{B}} T_{\mathrm{c}}$ has a shallow minimum; it approaches the BCS value for predominantly wide band electrons and surpasses it as the concentration of $d$-electrons increases above that of wide band electrons.

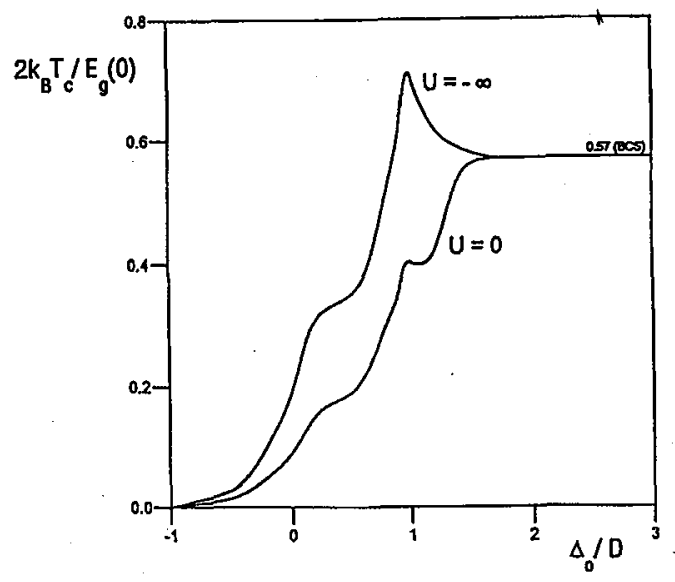

Fig. 7. $2 k_{\mathrm{B}} T_{\mathrm{c}} / E_{\mathrm{g}}(0)$ as a function of $\Delta_{0} / D$ for $n=1, I_{0} / 2 D=0.1, U \rightarrow-\infty$ and $U=0 . E_{\mathrm{g}}(0)$ denotes the gap in the single particle spectrum for $c$-electrons at $T=0$.

Decreasing $|U|$ yields a moderate reduction of $T_{\mathrm{c}}$ (up to a factor 2 at $\max T_{\mathrm{c}}$ if $U \rightarrow 0$ ), in comparison to the $U=-\infty$ case (cf. Fig. 6), and an analogous reduction of the ratio $k_{\mathrm{B}} T_{\mathrm{c}} / E_{\mathrm{g}}(0)$ in the (i) and (ii) regimes (cf. Fig. 7). On the contrary, in the (iii) regime $k_{\mathrm{B}} T_{\mathrm{c}} / E_{\mathrm{g}}(0)$ remains almost unaffected by $U$ and it approaches the BCS value (0.57) for any $U, \leq 0$ (comp. (A6) with (A8) and (A9) with (A10) in Appendix).

The SS-NO transition is continuous (2nd order) for any $U \leq 0$. As in the $J_{0} \neq 0, I_{0}=0$ case except for $U \rightarrow-\infty$, a nonzero fraction of nonpaired $d$-electrons exists above $T_{\mathrm{c}}$, but for substantial $|U|$ the $d$ pair density $n_{p}^{d}$ exhibits no sharp feature as $T$ is lowered through $T_{\mathrm{c}}$.

\subsection{Properties of the system for $U>0$}

For $U>0$ superconducting solutions $\left(x_{0} \neq 0, \rho_{0}^{x} \neq 0\right)$ of Eqs. (5) exist only below a definite value for $U\left(U_{0}\right)$, dependent on the interaction parameters and the temperature. Explicit expression determining $U_{0}$ at $T=0$ is given by 


$$
U_{0}=\frac{I_{0}^{2}}{2 N} \sum_{k}\left[\left(\varepsilon_{k}-\bar{\mu}\right)^{2}+\left(I_{0} \rho_{0}^{x}\right)^{2}\right]^{-1 / 2}
$$

and the plots of this quantity as a function of $n_{c} / n_{d}$ are shown in Fig. 8.

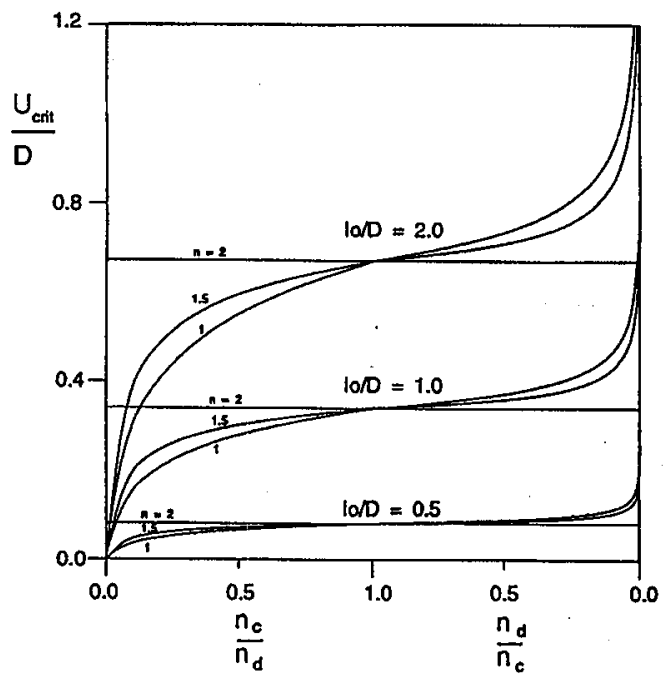

Fig. 8. The plots of $U_{c} / 2 D$ at $T=0$ as a function of $n_{c} / n_{d}\left(n_{d} / n_{c}\right)$ for fixed values of $n=n_{c}+n_{d}$ (numbers to the curves).

Obviously $U<U_{0}$ is only a necessary condition for the existence of superconductivity. The phase boundary between the SS and the normal state as a function of $U$ has to be found by a comparison of the free energies of both phases. One finds that at $T=0$ the transition to the normal phase occurring with increasing $U$ is always discontinuous (of the first order) and takes place at $U_{\mathrm{c}}<U_{0}$. The plot of the ground state phase boundary as a function of $U / 2 D$ and $I_{0} / 2 D$ for $n=2$ and $\Delta_{0} / D=1$ is shown in Fig. 9 .

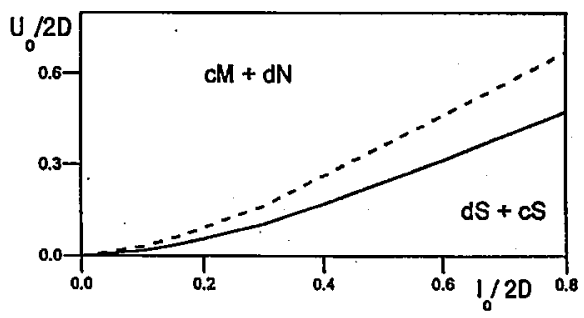

Fig. 9. The ground state phase boundary between the superconducting $(c S+d S)$ phase and normal $(c M+d N)$ phase for $n=2, \Delta_{0} / D=1$ as a function of $I_{0} / 2 D$ vs. $U / 2 D$. The transition to the normal phase is discontinuous (of the first order). Dashed line denotes the range of existence of the $\rho_{0}^{x} \neq 0, x_{0} \neq 0$ solutions. 


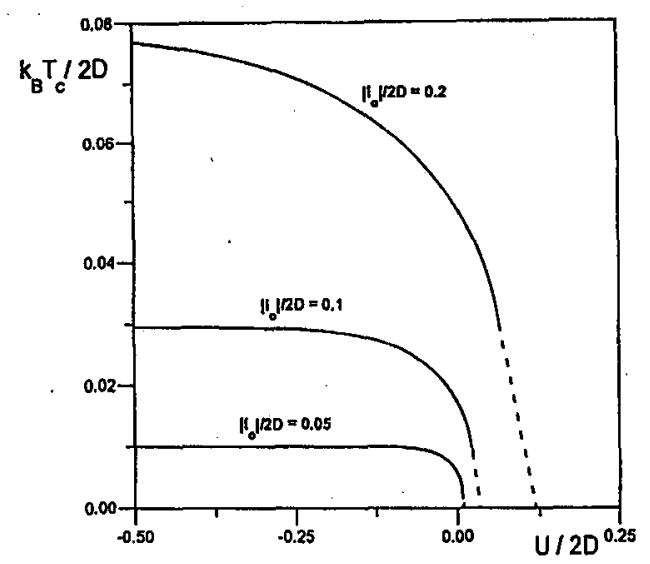

Fig. 10. The superconducting critical temperature as a function of $U / 2 D$ for three fixed values of $I_{0} / 2 D$ (numbers to the curves) and $n=2, \Delta_{0} / D=1$. The second- and first-order transitions are indicated by the solid and dashed lines, respectively.

Increasing repulsive $U$ reduces both the critical temperature and the ratio $k_{\mathrm{B}} T_{\mathrm{c}} / E_{\mathrm{g}}(0)$. In contrast to the attractive $U$ case, the $d$-pair density exhibits a sharp break at $T_{\mathrm{c}}$. For small $d$-pair binding energy (i.e. for substantial values of $U>0$ ) the critical temperature is determined by the $d$-pair breaking excitations and there are essentially no preformed pairs above $T_{\mathrm{c}}$.

For weak repulsion the SS transition at finite temperatures is continuous, as for $U<0$. However, increasing $U$ induces a change of the transition into the first order one. Finally, for $U>U_{c}$ the system remains in the normal state at any temperature. In Fig. 10 we show the finite temperature phase diagrams plotted as a function of $U / D$ for a few fixed values of $I_{0} / D\left(n=2, \Delta_{0} / D=1, J_{0}=0\right)$.

\section{Summary and concluding remarks}

In this paper we report results of the study of the phase diagrams and superconducting properties of the system made of coexisting itinerant $(c)$ electrons and localized $(d)$ electrons forming real space pairs. In contrast to previous works on the subject, which take as a starting point the fermion- (hard-core) boson models [1-10], the model considered' by us assumes arbitrary value of the on-site density interaction between localized electrons $U$, which allows investigation of the effects of reduced $d$-pair binding energy.

The main purpose of our work was to find the way the basic properties of the system change if this binding energy is reduced and what qualitatively new features can be introduced by the pair-breaking effects. Let us shortly summarize our findings.

We have analysed two types of mechanisms which can lead to the SS state in the system considered.

The first one is connected with the intersite charge exchange of $d$-electrons (pair hopping $J_{i j}$ ) and involves the narrow-band subsystem exclusively. In the limit 
of strong attraction of $d$-electrons, i.e. for large $d$-pair binding energy $E_{\mathrm{b}}$, it gives rise to a superconductivity analogous to superfluidity of hard-core charged bosons on a lattice [3] and the superconducting transition occurs from condensation of preexisting $d$-pairs. In the opposite regime, which is realised for substantial values of repulsive $U$, i.e. for small $E_{\mathrm{b}}$, the $\mathrm{SS}$ transition is determined by pair-breaking excitations and there are essentially no preformed pairs above $T_{c}$. It turns out that a crossover between the two regimes occurring with increasing $U$ is smooth and that above a critical value of $U$, dependent on concentration of $d$-particles, the SS state cannot be stable at any temperature.

The second mechanism involves the charge exchange between $d$ and $c$ electrons $\left(I_{0}\right.$ term) which leads to a superconducting state in both subsystems. For $I_{0} \neq 0$ the physical properties of the model strongly depend on the relative concentration of $d$ and $c$ particles. In particular, in the $d+c$ (mixed valence) regime the system shows features which are intermediate between those of pure local pair superconductor and those of classical BCS systems, even in the strong attraction case. This concerns, among others, the gap in the single-particle excitation spectrum for $c$-electrons, the ratio $k_{\mathrm{B}} T_{\mathrm{c}} / E_{\mathrm{g}}(0)$, the electromagnetic coherence length, the thermodynamic critical field as well as the properties of the normal state.

As we have stressed, in the $U \rightarrow-\infty$ limit the model considered reduces to that of coexisting fermions and hard-core bosons [1-3, 6-9]. Our analysis shows that several basic characteristics of the latter model, such as the general structure of phase diagrams and the evolution of $T_{c}$, the order parameters and the ratio $2 k_{\mathrm{B}} T_{\mathrm{c}} / E_{\mathrm{g}}(0)$ as a function of $\Delta_{0}$, are conserved for arbitrary $U<0$. Obviously, in contrast to the $U \rightarrow-\infty$ case, for any finite $d$-pair binding energy a nonzero fraction of nonpaired $d$-electrons exists above $T_{\mathbf{c}}$ and for low values of $|U|$ the $d$-pair density can exhibit a break as $T$ is lowered through $T_{\mathrm{c}}$.

The effects of repulsive $U$ are much more drastic as it competes with $I_{0}$ and $J_{i j}$. In both cases considered the repulsive $U$ can strongly reduce $T_{\mathrm{c}}$ and, for $I_{0} \neq 0$, the ratio $k_{\mathrm{B}} T_{\mathrm{c}} / E_{\mathrm{g}}(0)$. For a weak repulsion the superconducting transition remains continuous as for $U<0$, however the $d$-pair density exhibits a sharp break at $T_{\mathrm{c}}$. Increasing $U$ changes the nature of the phase transitions from a continuous to a discontinuous type, resulting in the tricritical point, and finally, for $U>U_{\mathrm{c}}$ the system remains in the normal state at any temperature.

Within our approach the $U$ term, which is the main factor determining the $d$-pair binding energy in the model, and the on-site $d$-density fluctuations, is treated exactly. Thus the major conclusions of the paper concerning the evolution of the properties of the system with $U$ are reliable for arbitrary $U$.

The mean-field (broken symmetry Hartree-Fock) approximations used for the intersite and intersubsystem interaction terms are best justified if these interactions are long-ranged. (Let us notice that for $I_{0}=0$ our treatment yields exact results in the thermodynamic limit for (i) $J_{i j}$ of infinite range: $J_{i j}=(1 / N) J$ for any $(i, j)$ - regardless dimensionality of the system [13] as well as for (ii) infinite-dimensional hypercubic lattice and nearest-neighbour $J_{i j}$.)

In the case of short-range $J_{i j}$ and $I_{0}$ interactions, however, this approach should be treated only as a standard first step in the investigation. Nonetheless, it is a useful guide for prediction of qualitative features of the phase diagrams even 
though the locations of critical points and shapes of the phase boundaries are not given precisely.

A better treatment of the low temperature thermodynamics including the collective excitation effects could be the self-consistent RPA-type approach, analogous to that used recently in the analysis of the pseudospin local-pair model [14]. For an improved description of the fluctuation effects in the critical region the modified Ginzburg-Landau theory [15] may be employed, as it has been done in Ref. [11] for the model Hamiltonian (2a). Both these investigations are left for future studies.

As in previous works, we assumed (for simplicity) the effective coupling $I_{0}$ to be $\left(k k^{\prime}\right)$ independent, which yields the $k$-independent gap parameter for $c$-subsystem. A study of the momentum-dependent intersubsystem charge exchange term, which could favour $d-, p$ - or extended $s$-types of pairing in $c$-subsystem would be of considerable interest [5], particularly in view of recent experimental results for cuprate superconductors [7].

There are several groups of materials for which the coexistence of local-pair states with itinerant electrons states have been either established or suggested and which can be suitable candidates for the present model [2, 3, 7, 16-20]. Some of these systems are nonstandard superconductors, other exhibit either a CDW state or they remain in the normal phase. A preliminary classification of these materials has been given in Ref. [2], whereas for a rather detailed analysis of the high $T_{\mathrm{c}}$ cuprates in this respect we refer the reader to Refs. [6-10].

Let us point out that the experimental results show the existence of $p$-holes in doped $\mathrm{BaBiO}_{3}\left(\mathrm{Ba}_{1-x} \mathrm{~K}_{x} \mathrm{BiO}_{3}\right.$ and $\left.\mathrm{BaPb}_{1-x} \mathrm{Bi}_{x} \mathrm{O}_{3}\right)$ [21], where the coexistence of interacting itinerant carriers and small bipolarons has been also indicated by the Raman spectroscopy data [20]. It suggests that realistic model for these materials should involve disproportionated (at least locally) $\mathrm{Bi}^{3+}-\mathrm{Bi}^{5+}$ background [22] coupled with oxygen $p$-holes.

\section{Acknowledgments}

We would like to thank R. Micnas, T. Kostyrko and J. Ranninger for many useful discussions.

This work was supported by the Committee for Scientific Research, project numbers: 2P302B 10411 and 2PO3B 05614.

Thanks are also due to the Polish-French cooperation through the Committee for Scientific Research-French Embassy agreement (Polonium).

\section{Appendix}

Below we present some analytical results concerning the critical temperatures, the ground state characteristics and the ratio $2 k_{\mathrm{B}} T_{\mathrm{c}} / E_{\mathrm{g}}(0)$, which can be derived in particular limits for the case $J_{0} \neq 0, I_{0} \neq 0$, using the rectangular density of states for $c$-electrons.

From Eqs. (5a, b, c) one obtains the following self-consistent equations for the second-order transition temperature $T_{\mathrm{c}}$ and the chemical potential:

$$
\frac{1}{2}\left(J_{0}+I_{0}^{2} S\right) B=1, \quad n-2=\tilde{\mu} B-G,
$$


where

$$
\begin{aligned}
& S=\frac{1}{2 N} \sum_{k} \frac{\tanh \beta\left(\varepsilon_{k}-\bar{\mu}\right)}{\varepsilon_{k}-\mu}, \\
& B=\frac{\sinh \beta_{c} \tilde{\mu}}{\widetilde{\mu}\left[\exp \left(\beta_{c} U / 2\right)+\cosh \beta_{c} \tilde{\mu}\right]}, \\
& G=\frac{1}{N} \sum_{k} \tanh \left[\frac{\beta_{c}\left(\varepsilon_{k}-\bar{\mu}\right)}{2}\right] .
\end{aligned}
$$

For the rectangular DOS for c-electrons (Eq. (8)):

$$
\begin{aligned}
S & =\frac{1}{4 D} \int_{0}^{2 D} d \varepsilon \frac{\tanh \left[\beta_{c} / 2(\varepsilon-\mu)\right]}{\varepsilon-\mu}, \\
. \quad G & =-\frac{1}{D \beta_{c}} \ln \frac{\cosh \left[\beta_{c}(2 D-\mu) / 2\right]}{\cosh \left(\beta_{c} \mu / 2\right)} .
\end{aligned}
$$

The energy gap in the single-particle spectrum for $c$-electrons at any $T<T_{\mathrm{c}}$ is given by

$$
\left.E_{\mathrm{g}}(T)=2 \min \left[\varepsilon_{k}-\bar{\mu}\right)^{2}+\left(I_{0} \rho_{0}^{x}\right)^{2}\right]^{1 / 2}
$$

where $\mu$ and $\rho_{0}^{x}$ have to be determined from Eqs. (5).

In general, $T_{\mathrm{c}}$ and $E_{\mathrm{g}}$ can only be found numerically and we have presented those results in Sec. 3 and 4. It is worthwhile to study the limiting cases which can be solved analytically.

The first two such cases corresponds to the limit of predominantly $c$-electrons with $d$ states either almost empty $\left(n_{d} \approx 0\right)$ or almost completely occupied $\left(n_{d} \approx 2\right)$ and they are realised for (i) $\Delta_{0} / D \gg n+J_{0} / 2 D$, if $n<2\left(n_{d} \approx 0\right)$ and for (ii) $\Delta_{0} / D \ll n-2-J_{0} / 2 D$, if $n>2\left(n_{d} \approx 2\right)$.

In the (i) case the chemical potential $\mu \approx n D$. From Eq. (A1), using the conditions $(2 D-\mu) \beta_{\mathrm{c}} \gg 1$ and $\mu \beta_{\mathrm{c}} \gg 1$, which are trivially satisfied, we obtain after straightforward calculations

$$
k_{\mathrm{B}} T_{\mathrm{c}}=\frac{2 \gamma}{\pi} D \sqrt{n /(2-n)} \exp \left(-\frac{\Delta_{0}-n D-J_{0} / 2}{I_{0}^{2} / D}\right),
$$

with $\gamma=\mathrm{e}^{C}, C=0.577$. In this lin it the energy gap at $T=0$ has the form

$$
E_{\mathrm{g}}(0)=2\left|I_{0} \rho_{0}^{x}\right|
$$

and the ground state value of $\rho_{0}^{x}$ calculated from Eqs. (5) for rectangular DOS is given by

$$
\rho_{0}^{x}=\frac{2 D}{\left|I_{0}\right|} \sqrt{n /(2-n)} \exp \left(-\frac{\Delta_{0}-n D-J_{0} / 2}{I_{0}^{2} / D}\right) .
$$

Equations (6) and (7) indicate an exponentially sharp dropoff of $T_{\mathrm{c}}$ and $E_{\mathrm{g}}(0)$ as $\Delta_{0} / D$ exceeds the characteristic value of $\Delta_{0}=n D+J_{0} / 2$, where practically all particles exist in $c$-band with only a small number of $d$-electrons present.

Analogous calculations performed for the (ii) case yield

$$
k_{\mathrm{B}} T_{\mathrm{c}}=\frac{2 \gamma}{\pi} D \sqrt{(n-2)(4-n)} \exp \left[-\frac{D(n-2)-\Delta_{0}-J_{0} / 2}{I_{0}^{2} / 4 D}\right] \text {, }
$$




$$
\rho_{0}^{x}=\frac{2 D}{\left|I_{0}\right|} \sqrt{(n-2)(4-n)} \exp \left[-\frac{D(n-2)-\Delta_{0}-J_{0} / 2}{I_{0}^{2} / D}\right] \text {. }
$$

As we see in both above cases the gap ratio takes a "classical" BCS value (cf. Fig. 7):

$$
2 k_{\mathrm{B}} T / E_{\mathrm{g}}(0)=\gamma / \pi \text {. }
$$

The next two cases correspond to the limit of predominantly $d$-electrons and very few $c$-electrons (or $c$-hole) realised for (iii) $\Delta_{0} / D \gg 0$, if $n<2\left(n_{c} \approx 0\right)$, and for (iv) $\Delta_{0} / D \ll 2 D$, if $n>2\left(n_{c} \approx 2\right)$.

In the (iii) case $\mu \approx \Delta_{0}$ and $n_{d} \approx n-(T / D) \exp \left(-\Delta_{0} / T\right)$. We now have $(2 \Delta-\mu) \beta_{\mathrm{c}} \gg 1$, and $-\mu \beta_{\mathrm{c}} \gg 1$, and from Eqs. (1) we obtain

$$
k_{\mathrm{B}} T_{\mathrm{c}}=\frac{r}{2}\left[J_{0}+\frac{I_{0}^{2}}{4 D} \ln \frac{2 D+\left|\Delta_{0}\right|}{\left|\Delta_{0}\right|} \frac{n-1}{\ln [n /(2-n)]}\right] \text {, }
$$

where $r=2$ for $U \rightarrow-\infty, r=1$ for $U=0$.

In the considered case the energy gap at $T=0$ is given by

$$
E_{\mathrm{g}}(0)=2\left[\bar{\mu}^{2}+\left(I_{0} \rho_{0}^{x}\right)^{2}\right]^{1 / 2},
$$

where $\mu$ and $\rho_{0}^{x}$ derived from Eqs. (5) are

$$
\bar{\mu}=\Delta_{0}+J_{0} \frac{n-1}{2}, \quad \rho_{0}^{x}=\frac{1}{2} \sqrt{n(2-n)} .
$$

Thus

$$
\frac{2 k_{\mathrm{B}} T_{\mathrm{c}}}{E_{\mathrm{g}}(0)}=\frac{r}{2} \frac{\left(J_{0}+\frac{I_{0}^{2}}{4 D} \ln \frac{2 D+\left|\Delta_{0}\right|}{\left|\Delta_{0}\right|}\right) \frac{n-1}{\ln [n /(2-n)]}}{\sqrt{\left|\Delta_{0}+J_{0}(n-1) / 2\right|^{2}+I_{0}^{2} n(2-n) / 4}} .
$$

Similar calculations for the (iv) case give

$$
\begin{aligned}
& k_{\mathrm{B}} T_{\mathrm{c}}=\frac{r}{2}\left[J_{0}+\frac{I_{0}^{2}}{4 D} \ln \frac{\left|\Delta_{0}\right|}{\left|\Delta_{0}\right|-2 D}\right] \frac{n-3}{\ln [(n-2) /(4-n)]}, \\
& E_{\mathrm{g}}(0)=2\left[(2 D-\mu)^{2}+\left(I_{0} \rho_{0}^{x}\right)^{2}\right]^{1 / 2}
\end{aligned}
$$

where

$$
\bar{\mu}=\Delta_{0}+J_{0} \frac{n-3}{2}, \quad \rho_{0}^{x}=\frac{1}{2} \sqrt{(n-2)(4-n)} .
$$

As we see from Eqs. (14), (15) in the limit of predominantly d-electrons $2 k_{\mathrm{B}} T / E_{\mathrm{g}}(0)$ is much lower than the BCS value. With increasing $\left|\Delta_{0}\right|$, this ratio is diminished, whereas $T_{\mathrm{c}}$ exhibits a slow dropoff towards a saturation value.

\section{References}

[1] J. Ranninger, S. Robaszkiewicz, Physica B 135, 468 (1985).

[2] S. Robaszkiewicz, R. Micnas, J. Ranninger, Phys, Rev. B 46, 180 (1987).

[3] R. Micnas, J. Ranninger, S. Robaszkiewicz, Rev. Mod. Phys. 62, 113 (1990).

[4] R. Friedberg, T.D. Lee, Phys. Rev. B 40, 6745 (1989); R. Friedberg, T.D. Lee, M.C. Ren, Phys. Rev. B 42, 4122 (1990); ibid. 45, 10732 (1992). 
[5] V.B. Geshkenbein, L.B. Ioffe, A.I. Larkin, Phys. Rev. B 55, 3173 (1997); C.P. Enz, Phys. Rev. B 54, 3589 (1996).

[6] Y. Bar-Yam, Phys. Rev. B 43, 359, 2601 (1991).

[7] R. Micnas, S. Robaszkiewicz, in: Ordering Phenomena in Condensed Matter Physics, Eds. Z.M. Galasiewicz, A. Pękalska, World Scientific, Singapore 1991, p. 127; R. Micnas, S. Robaszkiewicz, in: High-Tc Superconductivity 1996: Ten Years after the Discovery, Eds. E. Kaldis, E. Liarokapis, K.A. Müller, NATO ASI Series E, Vol. 342, Kluwer Academic Publ., Dordrecht 1997, p. 31 and references therein.

[8] I.O. Kulik, Int. J. Mod. Phys. B 2, 851 (1988); D.M. Eagles, Solid State Commun. 80, 823 (1991).

[9] B.R. Alascio, R. Allub, C.R. Proetto, C.I. Ventura, Solid State Commun. 77, 949 (1991); J.A. Wilson, Physica C 233, 332 (1994); Physica $C$ 182, 1 (1991); J.A. Wilson, A. Zahir, Rep. Prog. Phys. 60, 941 (1998).

[10] J. Ranninger, J.M. Robin, Phys. Rev. Lett. 74, 4027 (1995); Phys. Rev. B 53, 11961 (1996); T. Kostyrko, J. Ranninger, Phys. Rev. B 54, 13105 (1996).

[11] S. Robaszkiewicz, G. Pawłowski, Physica $C$ 210, 61 (1993); S. Robaszkiewicz, Acta Phys. Pol. A 85, 117 (1994).

[12] G. Pawłowski, S. Robaszkiewicz, Mol. Phys. Rep. 12, 191 (1995); W.R. Czart, M. Szkudlarek, S. Robaszkiewicz, Acta Phys. Pol. A 91, 415 (1997).

[13] R.A. Bari, Phys. Rev. B 7, 2128 (1973).

[14] A.S. Alexandrov, J. Ranninger, S. Robaszkiewicz, Phys. Rev. B 33, 4526 (1986); R. Micnas, S. Robaszkiewicz, Phys. Rev. B 45, 9900 (1992); R. Micnas, S. Robaszkiewicz, T. Kostyrko, Phys. Rev. B 52, 6863 (1995).

[15] L.N. Bulaevskii, V.L. Ginzburg, A.A. Sobyanin, Physica C 152, 378 (1988).

[16] E.K.H. Salje, in: Polarons and Bipolarons in High $T_{c}$ Superconductors and Related Materials, Eds. A.S. Alexandrov, W.Y. Liang, Cambridge University Press, Cambridge 1995, p. 110.

[17] J. Stanek, J. Chem. Phys, 76, 2315 (1982).

[18] N. Kojima, H. Kitagawa, T. Ban, F. Amita, M. Nakahara, Solid State Commun. $73,743(1990)$.

[19] R. Allub, B. Alascio, Solid State Commun. 85, 99 (1993).

[20] S. Sugai, Solid State Commun. 72, 1187 (1990); S. Sugai, Y. Enomoto, T. Murakami, Solid State Commun. 72, 1193 (1989).

[21] C.L. Lin, S.L. Qiu, Sie Chen, Myron Strongin, Gang Cao, Chan-Soo Iee, J.E. Crow, Phys. Rev. B 39, 9607 (1989).

[22] C.H. Rüscher, A. Heinrich, W. Urland, Physica C 219, 471 (1994); E.S. Hellman, E.H. Hartford, Jr., Phys. Rev. B 52, 6822 (1995). 